\title{
Magnetic Trapping of Hydrogen after Multistage Zeeman Deceleration
}

\author{
S. D. Hogan, A. W. Wiederkehr, H. Schmutz, and F. Merkt \\ Laboratorium für Physikalische Chemie, ETH Zürich, CH-8093, Switzerland
}

(Received 30 June 2008; published 29 September 2008)

\begin{abstract}
We report the first experimental realization of magnetic trapping of a sample of cold radicals following multistage Zeeman deceleration of a pulsed supersonic beam. $\mathrm{H}$ atoms seeded in a supersonic expansion of $\mathrm{Kr}$ have been decelerated from an initial velocity of $520 \mathrm{~m} / \mathrm{s}$ to $100 \mathrm{~m} / \mathrm{s}$ in a 12-stage Zeeman decelerator and loaded into a magnetic quadrupole trap by rapidly switching the fields of the trap solenoids.
\end{abstract}

DOI: 10.1103/PhysRevLett.101.143001

PACS numbers: $37.10 . \mathrm{Mn}, 32.60 .+\mathrm{i}$

Since the demonstration of magnetic trapping of lasercooled atoms [1] a range of techniques, involving the use of inhomogeneous electric or magnetic fields, have been developed to control the translational motion of, and confine neutral species which cannot be laser cooled. The goals of these techniques are to produce (ultra-)cold samples of a wide range of atoms and molecules, and to exploit such samples in a variety of applications such as precision spectroscopy, studies of molecular collisions at very low energies and of molecular gases close to quantum degeneracy. The present Letter describes the first experimental realization of magnetic trapping of a sample of cold radicals following adiabatic deceleration of a pulsed supersonic beam in a multistage Zeeman decelerator. Supersonic beams provide internally cold, dense $\left(10^{14}-10^{15} \mathrm{~cm}^{-3}\right)$ atomic or molecular samples with a high longitudinal velocity and a narrow longitudinal velocity spread. Adiabatic deceleration of such samples enables one to preserve the phase-space density from the source to the point where the sample is brought to rest in the laboratory frame.

Phase-stable multistage deceleration of pulsed supersonic beams of polar molecules was realized by Bethlem et al. using a multistage Stark decelerator [2,3]. This work led to electrostatic trapping of Stark-decelerated samples of ammonia at temperatures of $\sim 30 \mathrm{mK}$ [4] and to the determination of the lifetime of the metastable $a^{3} \Pi$ state of $\mathrm{CO}$ [5]. Polar $\mathrm{OH}$ molecules have also been loaded into electrostatic [6] and magnetoelectrostatic [7] traps following multistage Stark deceleration.

Rydberg Stark deceleration has also been used to decelerate $[8,9]$ and electrostatically trap samples excited to well-defined Rydberg states at temperatures of $\sim 150 \mathrm{mK}$ [10], while maintaining the phase-space density of the initially excited bunch. The development of the technique of multistage Zeeman deceleration [11-16], the magnetic analogue of multistage Stark deceleration has expanded the range of species which can be adiabatically decelerated to include all paramagnetic atoms and molecules. Multistage Zeeman deceleration can be added to the broad range of techniques now available for the production of cold molecules [17-22]. The ability to trap such species magnetically following deceleration presents several advantages for the applications listed above: (i) Radicals are reactive species and several radical-radical reactions are near barrierless and take place at very low temperatures [23], (ii) the electron spin of atomic and molecular radicals provides a quantized degree of freedom well suited for quantum information processing, and (iii) several cold paramagnetic atoms and molecules such as $\mathrm{H}$ and isotopomers, or $\mathrm{YbF}$, are ideal samples for measuring the Rydberg constant [24], the electric dipole moment of the electron [25], and the mass of the neutrino [15].

The 12-stage Zeeman decelerator employed in this work is a modified version of that reported previously [13]. $\mathrm{H}$ atoms are produced by $193 \mathrm{~nm}$ excimer laser photolysis of $\mathrm{NH}_{3}$ seeded in a $\mathrm{Kr}$ beam (a 10:1 Kr: $\mathrm{NH}_{3}$ gas mixture is used). Photolysis takes place in a quartz capillary $(1 \mathrm{~mm}$ inside diameter) mounted on the base plate of a pulsed valve (Even-Lavie Type E.L.-5-2005 [26]). The resulting $\mathrm{H}$ atom beam then propagates through a skimmer (orifice diameter $2 \mathrm{~mm}$ ) before entering the decelerator. The decelerator is composed of 12 solenoids wound on the outside of a quartz tube $(5 \mathrm{~mm}$ inside diameter, $7 \mathrm{~mm}$ outside diameter) which is immersed in cooling water. Each solenoid comprises 64 windings of $400 \mu \mathrm{m}$ enameled copper wire, wound in four layers, and is $7.3 \mathrm{~mm}$ long. The 12 solenoids are spaced on axis by $3.7 \mathrm{~mm}$. Currents of $300 \mathrm{~A}$ are pulsed in each solenoid to produce a maximal field of $2.2 \mathrm{~T}$ on axis. This field can be switched on and off with rise and fall times of $6 \mu \mathrm{s}$, and the decelerator is operated at a repetition rate of $10 \mathrm{~Hz}$ and a phase angle of $50^{\circ}$ [13]. To avoid Majorana transitions, the set of solenoids which form the decelerator are wired in a Helmholtz configuration and current pulses in adjacent solenoids are overlapped by $3 \mu$ s to maintain a nonzero field at the location of the decelerated bunch of $\mathrm{H}$ atoms as it propagates [12].

A magnetic quadrupole trap consisting of two coaxial solenoids is mounted at the end of the decelerator (see Fig. 1). The $\mathrm{H}$ atom beam exiting the decelerator enters the trap on axis through the solenoid labeled A. Both trap solenoids are composed of 72 windings of enameled copper tubing (340 $\mu \mathrm{m}$ inside diameter, $700 \mu \mathrm{m}$ outside diameter) wound in 4 layers. The innermost layer of 
(a)
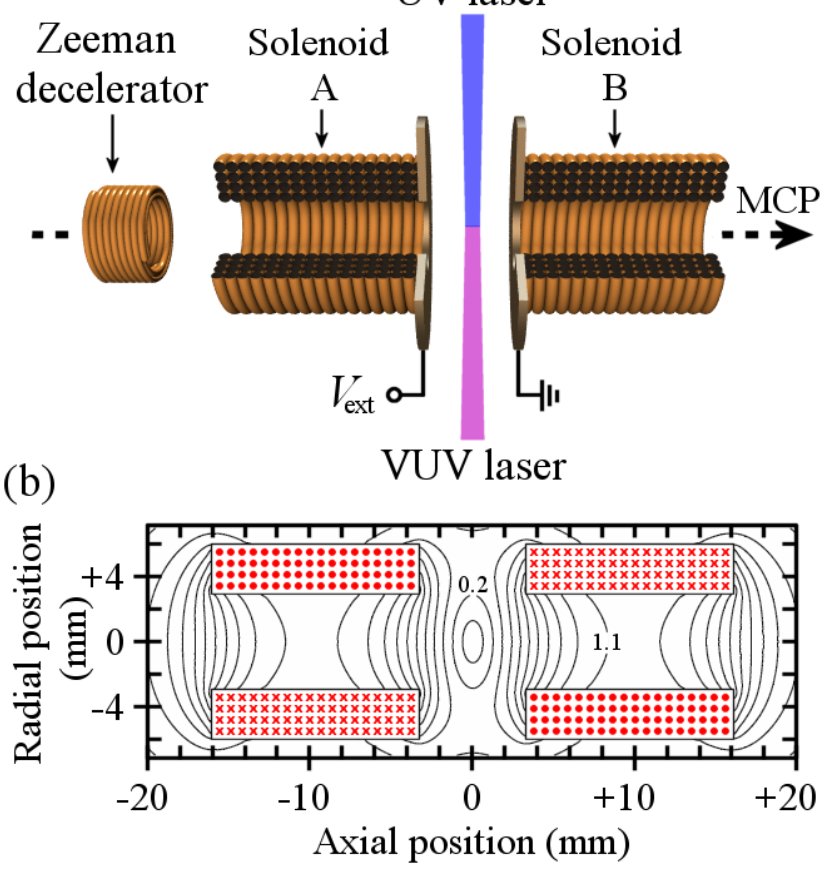

FIG. 1 (color online). (a) Schematic diagram of the quadrupole trap. The $\mathrm{H}$ atom beam propagates from left to right and after exiting the 12-stage decelerator enters the two solenoids (labeled $\mathrm{A}$ and B) which form the trap. Atoms in the trap volume are detected by VUV + UV two-photon excitation to Rydberg states followed by pulsed electric field ionization. $\mathrm{H}^{+}$ions are then extracted through solenoid B to the MCP detector (not pictured). (b) Distribution of magnetic field strength in the plane containing the axis of symmetry of the quadrupole trap for currents of $200 \mathrm{~A}$ applied to each solenoid. The contours of constant magnetic field strength are drawn in steps of $0.1 \mathrm{~T}$.

windings is wound at a radius of $3 \mathrm{~mm}$. The two solenoids are $13 \mathrm{~mm}$ long and are spaced by $6.6 \mathrm{~mm}$ on axis. Currents of $200 \mathrm{~A}$ are pulsed in each of these solenoids giving rise to maximal fields on axis of $\sim 1.2 \mathrm{~T}$. The solenoids are cooled by a constant flow $(\sim 4 \mathrm{ml} / \mathrm{min})$ of deionized water through the copper tubing. When operated at this current with pulses of $\sim 1 \mathrm{~ms}$ duration these trap solenoids can be cooled sufficiently to permit operation at a repetition rate of $10 \mathrm{~Hz}$. The distribution of magnetic field strength in the vicinity of the trap is displayed in Fig. 1(b). The field gradients surrounding the trap minimum are $\sim 1.2 \mathrm{~T} / \mathrm{cm}$, and the lowest saddle point is at a field strength of $0.24 \mathrm{~T}$ and corresponds to a trap depth of $130 \mathrm{mK}$ for $\mathrm{H}$ atoms (equivalent to a Zeeman shift of $0.09 \mathrm{~cm}^{-1}$ ).

Detection of $\mathrm{H}$ atoms near the trap center is carried out by two-photon excitation to Rydberg states via the $2^{2} P$ state using pulsed lasers operating at $121 \mathrm{~nm}$ (Lyman- $\alpha$ ) and $366 \mathrm{~nm}$. The excited atoms are then ionized by a pulsed electric field of $\sim 1.9 \mathrm{kV} / \mathrm{cm}$ and $160 \mathrm{~ns}$ duration applied between a pair of thin slotted copper disks located at the ends of the trap solenoids. The ions produced by pulsed electric field ionization are then accelerated on axis through solenoid B toward a microchannel plate (MCP) detector. By changing the delay between producing the $\mathrm{H}$ atoms and probing the trap volume, time-of-flight (TOF) profiles of the decelerated and trapped species can be obtained, and their velocity distributions determined [13].

Loading the quadrupole trap after multistage deceleration of the atoms in low-field seeking states to a velocity of $\sim 100 \mathrm{~m} / \mathrm{s}$, takes place in three stages labeled I, II, and III in Fig. 2. As the bunch of decelerated atoms exits the decelerator, solenoid A is switched on to overlap in time with the switch off of the last decelerator solenoid and thus to inhibit Majorana transitions [12] (stage I). The current applied is such that the direction of the magnetic field on axis at the center of this solenoid is the same as that of the decelerator solenoids. Solenoid A serves as a final deceleration stage before the $\mathrm{H}$ atoms enter the trap, and it provides axial compression of the decelerated bunch of atoms. The switch-off time of this initial current pulse is also chosen to partially transversally focus the decelerated bunch of atoms towards the trap center. The second stage of trap loading, II, involves switching on trap solenoid B just before solenoid A is switched off, again to avoid

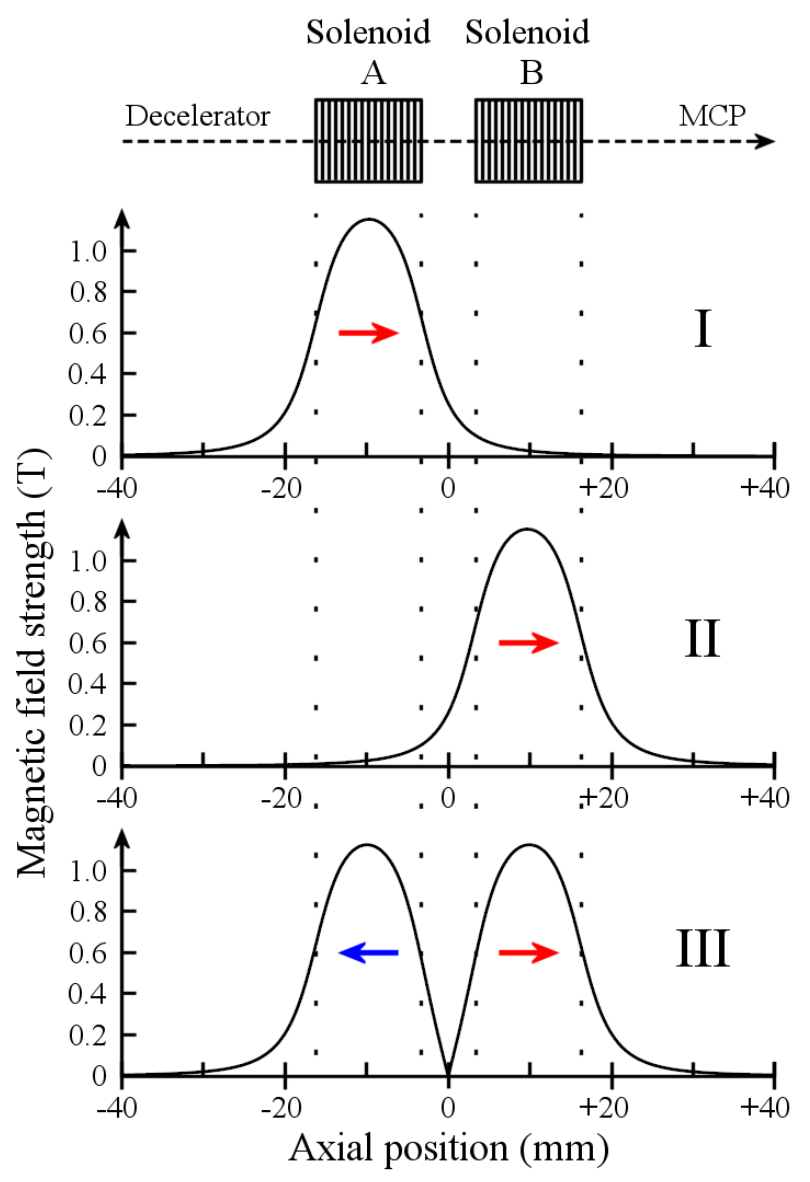

FIG. 2 (color online). Schematic diagram outlining the switching sequence employed when loading the quadrupole trap. The arrows indicate the direction of the magnetic field on axis at the center of the solenoid. 
Majorana transitions. The current applied to solenoid B gives rise to a magnetic field in the same direction as that resulting from the pulse applied to solenoid A in stage I. The decelerated bunch of atoms slows down further as it approaches solenoid B. When the center of the bunch is located at the midpoint between solenoids $\mathrm{A}$ and $\mathrm{B}$, solenoid A is switched on a second time with the current reversed. During this final stage of trap loading, III, the direction of the magnetic field in each of the solenoids is antiparallel resulting in the formation of a field distribution which traps the decelerated atoms in low-field-seeking quantum states [see Fig. 1(b)].

The traces in Fig. 3 demonstrate trapping using the procedure described. $\mathrm{H}$ atoms are decelerated from an initial longitudinal velocity of $520 \mathrm{~m} / \mathrm{s}$. The measured TOF profile of the $\mathrm{H}$ atom beam from the photolysis position to the center of the trap with the decelerator and trap turned off is displayed in Fig. 3(a). The beam has an initial velocity spread corresponding to a relative temperature of $\sim 1 \mathrm{~K}$. With the decelerator operating but not the trap solenoids, the TOF profile presented in Fig. 3(b) was obtained. The TOF profile exhibits three main features [13]. The sharp peak at a TOF of $0.63 \mathrm{~ms}$ corresponds to a bunch of atoms that are initially faster than the atoms for

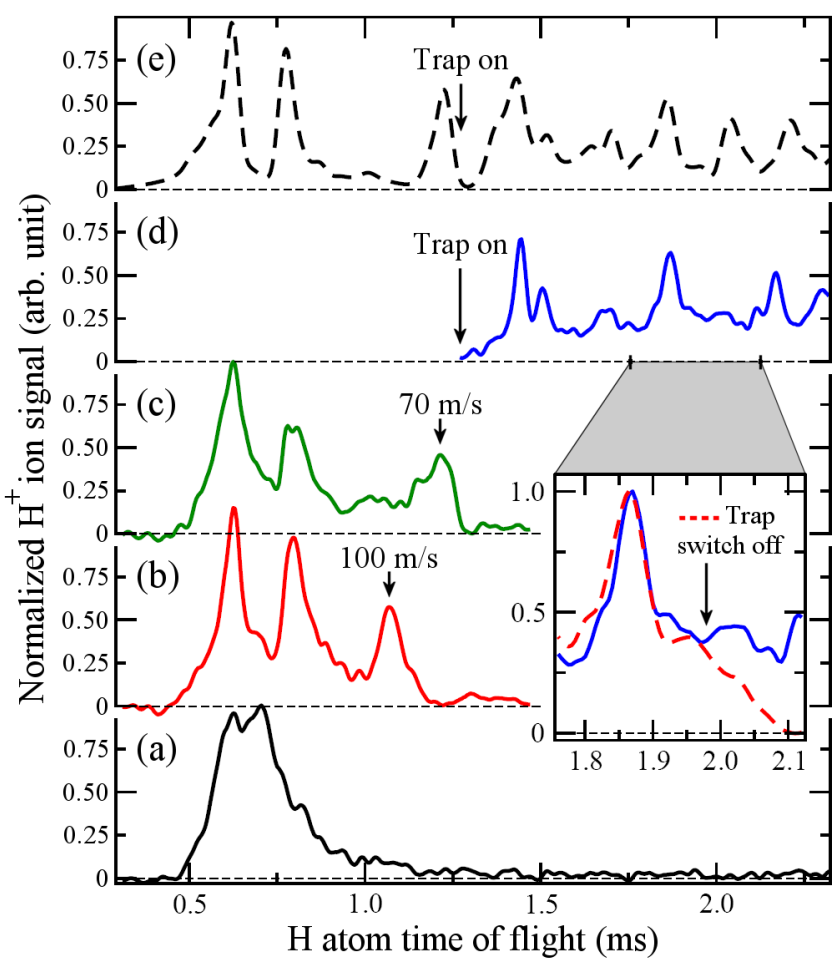

FIG. 3 (color online). TOF profiles of the $\mathrm{H}$ atom beam (a) with the decelerator off, (b) with the 12-stage decelerator on but with the trap solenoids off, (c) after deceleration with the decelerator and the first trap solenoid. (d) Detection of trapped atoms after switching on the trap. (e) Simulation of the experimental results. The inset is an expanded view of part of trace (d) along with the signal measured when the trap is switched off at $1.98 \mathrm{~ms}$ (dashed curve). which the deceleration pulse sequence was optimized. These atoms are transversely guided and accelerated in the first decelerator solenoids before they fall out of phase with the pulse sequence. The second feature at a TOF of $0.80 \mathrm{~ms}$ originates from a group of atoms with phase-space characteristics which differ from those of the decelerated atoms but are strongly transversely guided and slightly decelerated only in the last stages of the decelerator (see Ref. [13] for more details). The third feature, centered at $\sim 1.07 \mathrm{~ms}$, corresponds to the bunch of atoms for which the decelerator pulse sequence was optimized. This bunch of atoms has a mean final longitudinal velocity of only $100 \mathrm{~m} / \mathrm{s}$.

Using solenoid A to further slow the bunch of decelerated atoms from $100 \mathrm{~m} / \mathrm{s}$ to $70 \mathrm{~m} / \mathrm{s}$ (stage I in Fig. 2), the TOF profile displayed in Fig. 3(c) was measured. The bunch of decelerated atoms is shifted to a later arrival time of $1.21 \mathrm{~ms}$ and is also dispersed longitudinally as a result of the longer time taken to reach the detection point. After passing the center of the trap the decelerated atoms move up the wall of the trap towards solenoid B. However, they do not have sufficient kinetic energy to reach the point of maximal field strength and they are reflected and refocused towards the center of the trap. In stage II when only solenoid B is on, the strong field between the solenoids prohibits efficient detection of the $\mathrm{H}$ atoms. However, when both solenoids are on in the trapping configuration, III, the magnetic field strength close to the trap center, where detection takes place, is sufficiently low that Rydberg states can be efficiently excited. Consequently, the presence of trapped atoms can only be monitored reliably after solenoid A has been turned on for the second time. Trace (d) in Fig. 3 is therefore only displayed after $1.25 \mathrm{~ms}$. The reflected and refocused atoms give rise to the sharp feature at a time of $1.44 \mathrm{~ms}$. For this measurement, the trap remains on for a duration of $1 \mathrm{~ms}$ (limited by the current water cooling efficiency) over which time no decay of the trapped population is apparent. Several oscillatory refocusing events of the trapped bunch of atoms are observed during the first millisecond of trapping. These are gradually damped out as a result of the anharmonicity of the trapping potential.

To rule out the possibility that $\mathrm{H}$ atoms produced by photolysis of ammonia by the detection lasers or from desorption from the trap solenoids contribute to the trapped $\mathrm{H}$ atom signal in Fig. 3(d), the experiments were repeated (i) without the photolysis laser on and (ii) without pulsing the decelerator solenoids. In both cases the signal disappeared completely, indicating that all $\mathrm{H}$ atoms detected are produced by excimer laser photolysis and are decelerated before entering the trap. To further demonstrate trapping and to obtain an estimate of the temperature of the trapped cloud of atoms, experiments were also carried out in which the current in the trap solenoids was abruptly switched off (fall time of $10 \mu \mathrm{s}$ ) at a time of $1.98 \mathrm{~ms}$, i.e., after $700 \mu \mathrm{s}$ of trapping. The dashed line in the inset of Fig. 3 shows that under these conditions the $\mathrm{H}$ atom signal returned 
to zero within $100 \mu$ s. The full line also displayed was recorded without switching the trap off, and the fact that both traces in the inset exhibit the same structure up to $1.98 \mu \mathrm{s}$ demonstrates the reproducibility of these measurements. From the rate of signal loss after the trap is switched off and the size of the detection volume (this corresponds to a $5 \mathrm{~mm}$ long section of a cylinder with a diameter of $\sim 1 \mathrm{~mm}$, determined by the intersection of the VUV laser beam and the atom cloud) the temperature of the trapped atoms can be estimated to be $\sim 100 \mathrm{mK}$, though it is clear from the temporal structure of the $\mathrm{H}$ atom signal in Fig. 3(d) that the trapped cloud is not in equilibrium.

Presented in Fig. 3(e) are the results of a particle trajectory simulation from which the $\mathrm{H}$ atom signal at the detection position has been extracted throughout the deceleration and trapping process. The simulation is based on the same principles as those described in Ref. [13] and represents an important part of the optimization and validation of the experiments. The sharp features observed in the experimental data when the trap is on are convincingly reproduced, and in addition all of the main peaks in the TOF distributions are accounted for.

The spatial properties of the trap are imposed by the small size of the detection laser beams which necessitates a strong confinement for efficient detection, giving rise to some heating of the slow bunch of atoms exiting the decelerator. Both simulations and measurements of relative signal intensities show that the trapping efficiency is greater than 0.8 for the decelerated bunch of atoms. As the temperature of the cloud is $30 \mathrm{mK}$ at the exit of the decelerator [13], trap depths significantly lower than $130 \mathrm{mK}$ could confine the decelerated atom cloud as efficiently as is the case here. The trapping method presented here is more broadly applicable than the methods employed so far to trap $\mathrm{H}$ atoms $[27,28]$ and can be used to trap all paramagnetic atoms and molecules, in particular, also $\mathrm{D}$ and $\mathrm{T}$ atoms which have not been trapped magnetically so far.

We thank M. Andrist, B. Lambillotte, and R. Gunzinger for their technical assistance. This work is supported by the Swiss National Science Foundation.

[1] A. L. Migdall, J. V. Prodan, W. D. Phillips, T. H. Bergeman, and H. J. Metcalf, Phys. Rev. Lett. 54, 2596 (1985).

[2] H. L. Bethlem, G. Berden, and G. Meijer, Phys. Rev. Lett. 83, 1558 (1999).

[3] H. L. Bethlem and G. Meijer, Int. Rev. Phys. Chem. 22, 73 (2003).

[4] H. L. Bethlem, F. M. H. Crompvoets, R. T. Jongma, S. Y. T. van de Meerakker, and G. Meijer, Phys. Rev. A 65, 053416 (2002).
[5] J. J. Gilijamse, S. Hoekstra, S. A. Meek, M. Metsälä, S. Y. T. van de Meerakker, and G. Meijer, J. Chem. Phys. 127, 221102 (2007).

[6] S. Y. T. van de Meerakker, P. H. M. Smeets, N. Vanhaecke, R. T. Jongma, and G. Meijer, Phys. Rev. Lett. 94, 023004 (2005).

[7] B. C. Sawyer, B. L. Lev, E. R. Hudson, B. K. Stuhl, M. Lara, J.L. Bohn, and J. Ye, Phys. Rev. Lett. 98, 253002 (2007).

[8] S. R. Procter, Y. Yamakita, F. Merkt, and T.P. Softley, Chem. Phys. Lett. 374, 667 (2003).

[9] E. Vliegen and F. Merkt, J. Phys. B 38, 1623 (2005).

[10] S. D. Hogan and F. Merkt, Phys. Rev. Lett. 100, 043001 (2008).

[11] N. Vanhaecke, U. Meier, M. Andrist, B. H. Meier, and F. Merkt, Phys. Rev. A 75, 031402(R) (2007).

[12] S. D. Hogan, D. Sprecher, M. Andrist, N. Vanhaecke, and F. Merkt, Phys. Rev. A 76, 023412 (2007).

[13] S. D. Hogan, A. W. Wiederkehr, M. Andrist, H. Schmutz, and F. Merkt, J. Phys. B 41, 081005 (2008).

[14] E. Narevicius, C. G. Parthey, A. Libson, J. Narevicius, I. Chavez, U. Even, and M. G. Raizen, New J. Phys. 9, 358 (2007)

[15] E. Narevicius, A. Libson, C. G. Parthey, I. Chavez, J. Narevicius, U. Even, and M. G. Raizen, Phys. Rev. Lett. 100, 093003 (2008).

[16] E. Narevicius, A. Libson, C. G. Parthey, I. Chavez, J. Narevicius, U. Even, and M. G. Raizen, Phys. Rev. A 77, 051401(R) (2008).

[17] J. M. Doyle, B. Friedrich, J. Kim, and D. Patterson, Phys. Rev. A 52, R2515 (1995).

[18] S. E. Maxwell, N. Brahms, R. deCarvalho, D. R. Glenn, J. S. Helton, S. V. Nguyen, D. Patterson, J. Petricka, D. DeMille, and J.M. Doyle, Phys. Rev. Lett. 95, 173201 (2005).

[19] M. S. Elioff, J. J. Valentini, and D. W. Chandler, Science 302, 1940 (2003).

[20] S. A. Rangwala, T. Junglen, T. Rieger, P. W. H. Pinkse, and G. Rempe, Phys. Rev. A 67, 043406 (2003).

[21] A. Fioretti, D. Comparat, A. Crubellier, O. Dulieu, F. Masnou-Seeuws, and P. Pillet, Phys. Rev. Lett. 80, 4402 (1998).

[22] S. Willitsch, M. T. Bell, A. D. Gingell, S. R. Procter, and T. P. Softley, Phys. Rev. Lett. 100, 043203 (2008).

[23] I. R. Sims and I. W. M. Smith, Annu. Rev. Phys. Chem. 46, 109 (1995).

[24] A. Huber, B. Gross, M. Weitz, and T. W. Hänsch, Phys. Rev. A 59, 1844 (1999).

[25] M. R. Tarbutt, H. L. Bethlem, J. J. Hudson, V. L. Ryabov, V. A. Ryzhov, B. E. Sauer, G. Meijer, and E. A. Hinds, Phys. Rev. Lett. 92, 173002 (2004).

[26] M. Hillenkamp, S. Keinan, and U. Even, J. Chem. Phys. 118, 8699 (2003).

[27] I. F. Silvera and J. T. M. Walraven, Phys. Rev. Lett. 44, 164 (1980).

[28] R. W. Cline, D. A. Smith, T. J. Greytak, and D. Kleppner, Phys. Rev. Lett. 45, 2117 (1980). 